\title{
EJNSO
}

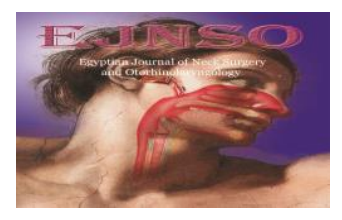

\section{Botulinum Toxin injection in Bilateral Vocal Fold Immobility}

\author{
Reham AE Ibrahim ${ }^{1}$, Mohamed M. Aboshanif ${ }^{2}$, Shereen Farghaly ${ }^{3}$
}

1. Department of Otorhinolaryngology, Phoniatrics unit, Faculty of Medicine, Assiut University, Assiut, Egypt.

2. Department of Otorhinolaryngology, Faculty of Medicine, Assiut University, Assiut, Egypt.

3. Department of Chest Diseases, Assiut University, Assiut, Egypt.

\section{Abstract:}

Introduction: Bilateral vocal fold paralysis (BVFP) is a complicated lifethreatening clinical problem. Airway enlargement procedures for bilateral vocal fold immobility, such as cordotomy and arytenoidectomy, permanently impair voice and increase the risk of aspiration. An innovative strategy for restoring ventilation without affecting voice or swallowing is botulinum toxin injection into the thyroarytenoid muscle.

Objective: To investigate the effectiveness of thyroarytenoid muscle chemical denervation with botulinum toxin injection in diminishing dyspnea in BVFP by changing the positioning of the paralyzed VFs.

Patients and Methods: 12 patients suffering from bilateral vocal folds immobility were enrolled in the study. Botulinum toxin injection was performed into each vocal fold via a per-oral approach. Voice evaluation, laryngoscopic examination, and pulmonary function tests were carried out before and 15 days after the injection .

Results: All patients reported improvement of their respiratory symptoms as well as improvement of the pulmonary function studies following the injection. Patients who received repeated injections demonstrated a longer duration of symptoms improvement. Voice handicap index scores were not significantly changed after botulinum toxin injection into each vocal fold.

Conclusion: Botulinum toxin injection is a less invasive, short term treatment option improving ventilation in patients with BFVP with little or no effect on voice or swallowing.

Keywords: Bilateral vocal fold immobility; Botulinum toxin; Thyroarytenoid muscles.

\section{$\underline{\text { Introduction }}$}

Bilateral vocal fold immobility (BVFI) is a serious problem, leading to narrowing of the glottis and causing stridor. It may be due to neurological insult, ankylosis of the cricoarytenoid
(CA) joint, or fibrosis of the posterior glottis. ${ }^{1}$

Recurrent laryngeal nerve injury may be of central or peripheral origin; central causes such as hydrocephalus, amyotrophic lateral sclerosis (ALS), 
upper motor neuron lesion, or closedhead trauma, while, peripheral causes often include viral neuritis, tumor, thyroidectomy or spine surgery. Common muscle diseases such as myasthenia gravis and muscular dystrophy may affect the laryngeal muscles leading to vocal fold immobility. $^{2}$

BVFI with stridor should be managed by tracheostomy to improve the airway. Nevertheless, there are many options to statically improve the airway and avoid or reverse tracheostomy have been used. These interventions create a balance between preserving the voice and achieving an adequate airway. They include cordotomy, arytenoidectomy, partial arytenoidectomy or medial arytenoidectomy, suture lateralization of the vocal fold, and lateral type II thyroplasty. $^{3}$

Some patients will suffer difficulty in breathing later on after their initial injury. This could be due to aberrant reinnervation resulting in synkinesis of the laryngeal muscles. Simultaneous activation of both abductors and adductors may result in improper contraction. In recent years, botulinum toxin injection has been used in patients with laryngeal synkinesis. ${ }^{4}$

Botulinum toxin $A$ acts at the neuromuscular junction by inhibiting acetylcholine release, hence paralyzing the muscle. It was first used in the larynx for the treatment of spasmodic dysphonia, laryngeal dystonia, or neurologic laryngeal dysfunction in $19866^{5}$ Further clinical studies revealed the relief of airway compromise when Onabotulinum toxin A was injected into the adductors of the larynx, weakening the force of laryngeal closure. This would rebalance the laryngeal muscles and adjust the arytenoid muscle by using the unopposed pull of the abductors. ${ }^{6}$
According to Woo and Mangaro, 2004, who reported that in some patients with unilateral RLN injury, this reinnervation could be a cause of stridor and laryngospasm. In two patients, they reported relief of these symptoms by chemodenervation of the affected vocal fold with botulinum toxin. ${ }^{7}$

Human trials reporting the effectiveness of chemical denervation of the adductor muscles in cases of bilateral vocal fold paralysis are limited. This study was designed to focus on the benefit of botulinum toxin injection into the thyroarytenoid muscle as one of the temporary means of management of synkinetic larynx in such cases.

\section{Patients and Methods:}

Study design: Prospective human clinical trial

Subjects: This study included 12 patients suffering from bilateral vocal folds immobility, with 2 of them had a previously performed tracheostomy at out Ear Nose and Throat Department. Patients were nine females and three males with an age range of 32-68 years. All patients showed no signs of improvement in their vocal fold mobility after at least 6-months of follow-up.

All patients underwent the following procedures:

I.Elementary diagnostic procedures: Patient interview and history taking; includes personal history, patient complaint, analysis of symptoms, and impact of the complaint on the patient by asking the patient to fill in the Voice Handicap Index (VHI) ${ }^{8}$ Arabic version, auditory perceptual assessment of patient's voice using the modified GRBAS scale ${ }^{9}$, general examinations, E.N.T. examination, type of breathing and head position, laryngeal examination; including 
external laryngeal examination and elementary visual impression and mirror laryngoscopy.

II. Clinical diagnostic aids: We have performed augmentation and documentation of the glottis picture and glottis gap subjectively using either $90^{\circ}$ rigid Tele-laryngoPharyngoscope- Hopkins ${ }^{\circledR}$ or flexible Video Rhino-Laryngoscope, color systems PAL/NTSC both endoscopies were connected to TELE PACK $\mathrm{X}$ LED TP 100 and a camera system Xion $\mathrm{CH}$ 01-D for the rigid one. As well as voice recording during prolonged /ah/ sound.

III.Respiratory evaluation: pulmonary function test was done using a spirometer (CosmedSrL, Quark PFTs ergo, P/N Co9035-12-99, made in Italy) to detect the peak inspiratory flow (PIF) rate. This measurement was taken through the mouth with the patient's nose, and tracheostomy site (if any) occluded immediately before and at least 15 days after the procedure. Results were expressed in liter and liter/second and as a percentage of normal value for gender, age, and height (percent predicted) and will be interpreted according to the American Thoracic Society/European Respiratory Society guidelines. ${ }^{10}$

$I V$.Procedure: Botulinum toxin $\mathrm{A}$; the toxin is reconstituted with $2.5 \mathrm{ml}$ saline with an initial dose of 2.5 IU into each vocal fold, and then the dose was increased to 5 IU injected bilaterally via a per-oral approach into the thyroarytenoid muscles. Injections were done primarily under general anesthesia in 10 patients using the traditional microscopic suspension laryngoscopy and 24gauge x0.394 ENT Needle-Double Bevel (12-000-00-ND1). Only two patients had their injection in the office setting using local anesthetic spray (Xylocaine 4\%) to apply topical anesthesia of the oropharynx and then through the working channel of a flexible laryngoscope; the laryngoscope is used for dripping $2 \%$ lidocaine onto the base of the tongue, epiglottis, and, while the patient is phonating, onto the vocal folds and then the toxin is deposited into the muscle via a curved injection needle and a $90^{\circ}$ rigid laryngoscope connected to a video-camera for visualization and monitoring of the injection.

Patients were followed up two weeks, one month, three months, and six months after the injections using the assessment mentioned above.

\section{Ethical considerations}

Our study was approved by the ethical committee of our medical faculty under number 17300139.

The aim of the study, as well as the investigation and surgical procedures, were explained to each patient before the procedure. Written consents were obtained from those who welcomed to participate in the study. Privacy and confidentiality of all data were assured.

\section{Statistical analysis :}

Data entry and data analysis were done using SPSS version 24 (Statistical Package of Social Science). Data were presented as number, percentage, mean, and standard deviation. Chi-square and Fisher Exact tests were used to compare qualitative variables. An independent sample t-test was used to compare quantitative variables between groups. P-value considered statistically significant when $\mathrm{P}<0.05$. A Paired-T test was used to distinguish between variables in the same group. 


\section{$\underline{\text { Results: }}$}

Twelve patients were enrolled in this study, nine females and three males with an age range of 32-68 years and a mean age of $45.83 \pm 11.67$. Eleven patients $(91.7 \%)$ had bilateral vocal folds immobility, and one patient $(8.3 \%)$ had bilateral vocal folds motion impairment. Post-thyroidectomy recurrent laryngeal nerve injury was the etiology in ten patients $(83.3 \%)$, idiopathic origin was found in one patient $(8.3 \%)$, and one patient $(8.3 \%)$ had bilateral vocal fold motion impairment due to Hashimoto $\mathrm{s}$ thyroiditis. Ten patients $(83.3 \%)$ were not tracheostomized, and only two (16.7\%) had a tracheostomy due to stridor before the start of our procedure, Table 1.

Table1: Demographic data of patients:

\begin{tabular}{|l|c|}
\hline \multicolumn{1}{|c|}{ Item } & Descriptive \\
\hline 1- Age "yrs." "mean \pm SD" & $45.83 \pm 11.67$ \\
2- Sex: & $3(25.0 \%)$ \\
- Male & $9(75.0 \%)$ \\
- Female & $10(83.3 \%)$ \\
3- Etiology of paralysis: & $1(8.3 \%)$ \\
- Thyroidectomy & $1(8.3 \%)$ \\
- Hashimoto,s Thyroiditis & \\
- Idiopathic & $11(91.7 \%)$ \\
4- Diagnosis of the paralysis: & $1(8.3 \%)$ \\
- Bilateral VF paralysis & \\
- Bilateral VF paresis & $2(16.7 \%)$ \\
5- Tracheostomy status: & $10(83.3 \%)$ \\
- Yes & \\
- No & \\
\hline
\end{tabular}

Four of the patients had single botulinum toxin injection, and the remaining eight patients had multiple injections with a mean number of injections $2 \pm 0.25$. Figure (1) demonstrates the relation between the number of injections and the duration of symptomatic improvement. There was a significant relationship between the two parameters with $r=0.737$ and $p=0.02$; patients with repeated injections demonstrated a longer duration of improvement in their respiratory symptoms. The mean duration of symptomatic improvement was $3.42 \pm$ 0.26 months. The four patients who had only a single botulinum toxin injection had reported improvement in their breathing on the first follow up visit and didn't show up for further appointments.

A pulmonary function test was done for all patients before and 15 days after the injection. The peak inspiratory flow (PIF) measurements were the primary interest. Data showed that there was a significant increase in the PIF following botulinum toxin injection with $\mathrm{p}=$ 0.000 , figure (2).

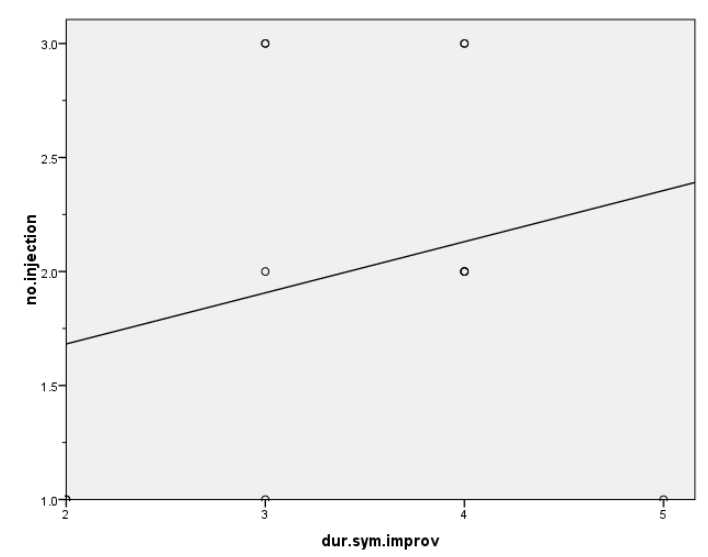

Figure 1: Relation between the number of injections and the duration of symptomatic improvement

Also, their measurements 1 and 3 months after the injection revealed the same increase with $\mathrm{p}=0.000$ and 0.01 , respectively. However, PIF measurements six months after the injection didn't show much increase yet, values were getting closer to the preinjections ones, figure (3). Another measurement was the peak expiratory flow (PEF) which was also noted to be increased following the injection yet, not significantly. 


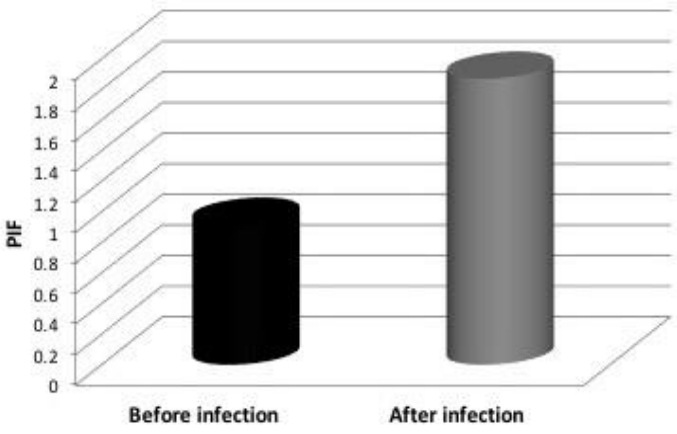

Figure 2: Peak inspiratory flow (PIF) measurement before and 15 days after botulinum toxin injection

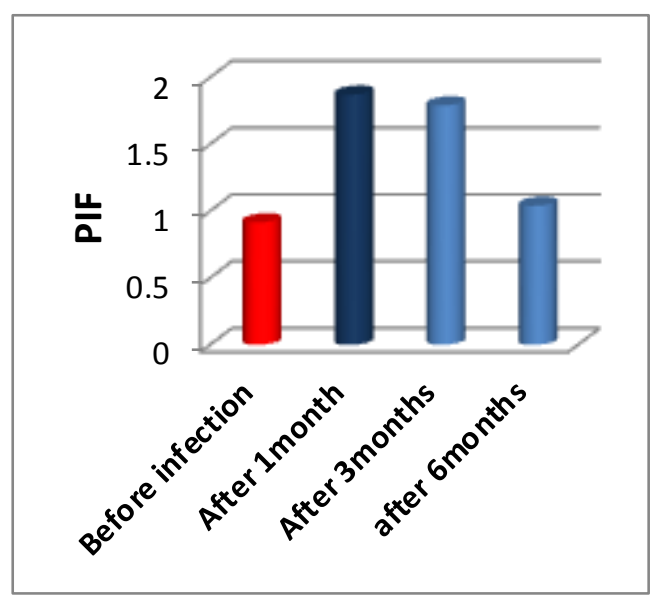

Figure 3: Peak inspiratory flow (PIF) measurement before and 1,3 \& 6 months after botulinum toxin injection.

Two of the patients had tracheostomy before botulinum toxin treatment, and we were able to close their tracheostomies ten days after the injection. Only one patient of the nontracheostomized group required tracheostomy immediately after the injection, which was decannulated three days later. Figure 4 shows the widening of the glottis gap two weeks after injection.

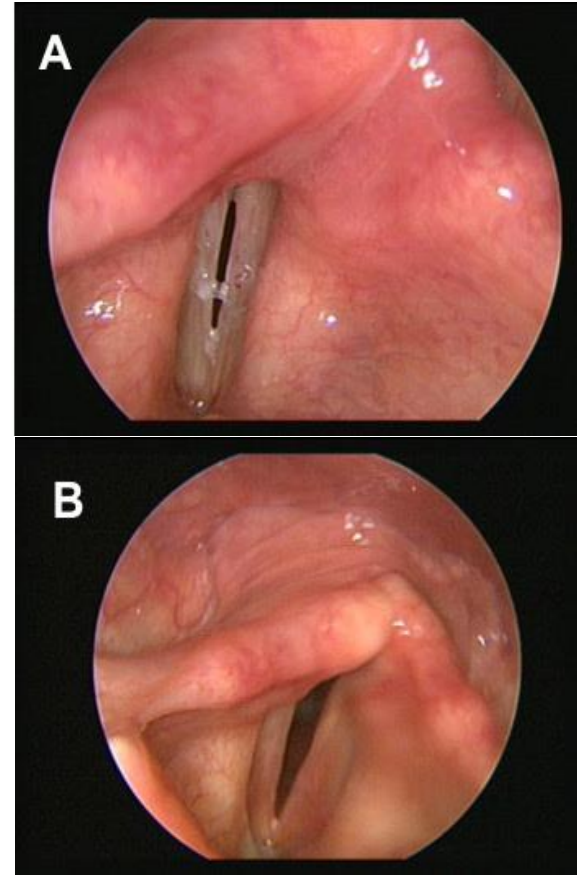

Figure 4: Laryngoscopic view of the vocal fold; (a) before botulinum toxin injection and (b) two weeks following the injection. Notice the slight increase of the glottis gap in (b).

Patients were divided into three groups according to the duration of paralysis.

Group 1: 6 months -1 year.

Group 2: 1 year -2 years.

Group 3: > 2 years.

Group 1 included six patients (50\%), and both groups 2 and 3; each included three patients (25\%). Analysis of data showed that patients who had a longer duration of paralysis required repeated injections, as shown in figure 4.

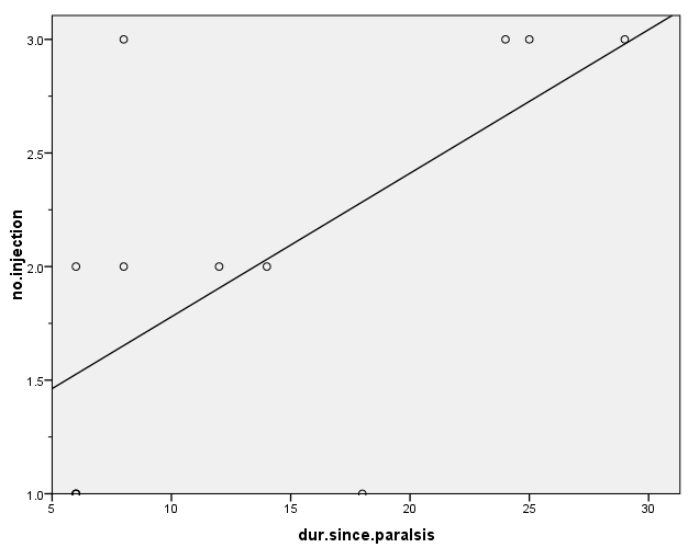

Figure 5: Relation between duration since paralysis and the number of botulinum toxin injections. 
Also, they needed a longer time to show manifestations of initial improvement in their breathing after injection, as shown in figure 6 .

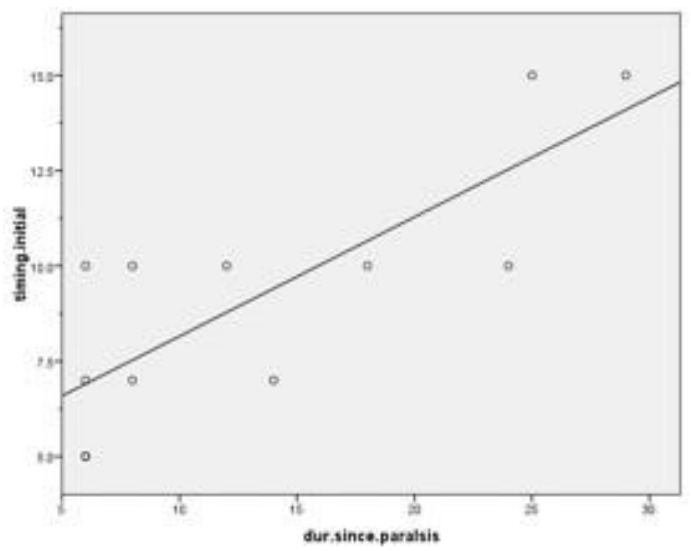

Figure 6: Relation between duration since the paralysis and timing of initial symptomatic improvement after repeated injection.

Voice evaluation was done before the start of the procedure, and 15 days later. Voice handicap index (VHI) scores were also calculated. Data showed that there were no significant changes in the VHI before and after the injection, as shown in figure 7.

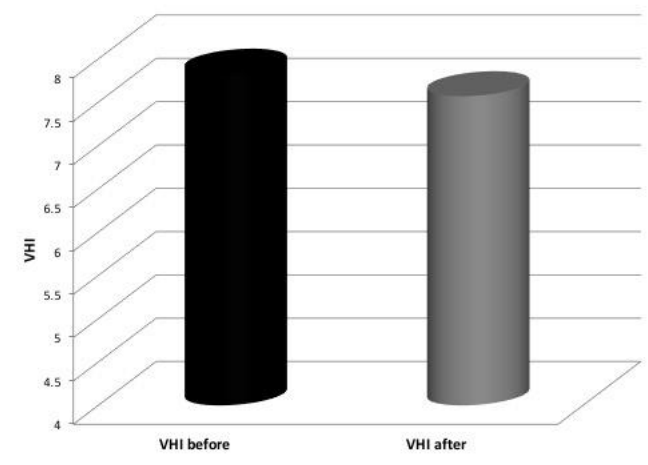

Figure 7: Relation between the Voice Handicap Index (VHI) before and after botulinum toxin injection.

\section{Discussion :}

Bilateral abductor paralysis is a common and often life-threatening clinical problem. There are many procedures for the widening of the glottic chink, such as cordotomy and arytenoidectomy, which permanently impair voice and increase the risk of aspiration. An innovative strategy for restoring ventilation without affecting voice or swallowing is botulinum toxin injection into each vocal fold. During the non-inspiratory phase, the vocal folds passively relax to the midline to allow for normal voicing and airway protection. $^{11}$

As the motor fibers in the recurrent laryngeal nerve (RLN) innervate both abductor and adductor muscles of the vocal fold, damage to the RLN will compromise both abduction and adduction functions. In most of these patients, random regeneration of neural fibers results in aberrant reinnervation of laryngeal muscles. In such a synkinetic larynx, contraction of both abductors and adductors produces ineffective, unsynchronized or even opposite movement of the vocal fold(s). ${ }^{12}$

Three to nine months after RLN injury, cross reinnervation between the adductor and abductor groups develops. So, In RLN firing, both groups are activated resulting in more narrowing of the airway because the thyroarytenoid and lateral cricoarytenoid (LCA) complex, which is responsible for vocal cords adduction. ${ }^{13}$

Daniel et al. 2014 tried to inject Botox into cricothyroid muscles through an external approach to oppose its action. ${ }^{14}$ On the contrary, in our study, we attempted to paralyze the thyroarytenoid muscles. The primary objective of this study was to improve ventilation in patients with bilateral abductor paralysis using chemical denervation of the thyroarytenoid muscles with botulinum toxin injection without sacrificing the voice or swallowing. 
There are limited human trials investigated the therapeutic effect of botulinum toxin in these cases, and that is why this study was conducted to explore its potential benefit.

Although laryngeal electromyography (EMG) can be helpful in the differentiation of vocal fold paralysis, vocal fold fixation, or arytenoids subluxation by showing abnormal EMG activation in VF paralysis. $15 \mathrm{We}$ depended upon the arytenoids palpation during direct laryngoscopy to exclude arytenoid fixation.

Almost all patients in our study demonstrated clinical improvement in their respiratory symptoms from botulinum toxin injection. This could be explained by the fact that chemical denervation of the thyroarytenoid muscles diminishes their passive tonus caused by aberrant reinnervation, allowing for the reinnervated abductors to produce an unopposed effect on vocal folds position. These data came in concordance with the results of a study by Ekbom et al., 2010 who reported improvement of 10 of their 11 patients following botulinum toxin injection into the thyroarytenoid/ lateral cricoarytenoid complex. ${ }^{6}$

There were significant increases in the peak inspiratory flow (PIF) measurements recorded 15 days after the injection amongst all patients; $p=0.000$ which was reflected on the potential improvement of the respiratory symptoms reported by the patients and this might be explained by decreasing the tonus of the adductor muscles in response to the injection. With regular follow-up at 1 and 3 months after injection, there was a statistically significant increase in the peak inspiratory flow with $\mathrm{p}=0.000$ and 0.01 , respectively. Our results were initially close to that observed by Zealear et al., 2002 who used Botox injection in combination with laryngeal pacing to stimulate PCA electrically. ${ }^{11}$

However, our PIF measurements 6 months after the injection showed a decline close to the pre-injections values. This is due to the temporary effect of botulinum toxin injection.

Another measurement of the pulmonary function is the peak expiratory flow (PEF), which was noted to be also increased following the injection. This may be caused by decreased glottis constriction during expiration, allowing for increased airflow during voicing that might explain the return of voice or the nonsignificant change in voice and VHI scores after injection.

In the current study, patients with repeated injections exhibited a longer duration of improvement in their respiratory symptoms with a mean duration of $3.42 \pm 0.26$ months. The initial dose of botulinum toxin was 2.5 IU into each vocal fold then the dose was increased to $5 \mathrm{IU}$ per each vocal fold. This increase in the dose was dependent on the duration of symptomatic improvement after each injection. This came to a disagreement with a study by Ekbom et al., 2010, who reported improvement of the respiratory symptoms in 10 of their 11 patients with only $2.5 \mathrm{IU}$ of botulinum toxin per vocal fold. This might be explained by the delayed commencement of botulinum toxin injection therapy in our cases as all of them received the injection six months after the onset of the disease. ${ }^{6}$

Most of our patients (50\%) presented with a duration of paralysis between 6 months to 1 year with a mean duration of paralysis about $13.50 \pm 2.45$ months. On the correlation between the duration of paralysis and the start of initial symptomatic improvement, data showed that patients with a longer duration of paralysis needed a longer time to 
demonstrate manifestations of initial improvement in their breathing after the injection. Perhaps due to a stronger degree of residual muscular activity in the reinnervated adductors that developed with time.

Two of our patients had tracheostomy before the procedure, and we were able to close their tracheostomies ten days after the injection. Only one patient required tracheostomy immediately after the injection as she showed manifestations of airway obstruction upon recovery from anesthesia, and she was decannulated three days after the injection.

Only 2 of our patients complained from mild dysphagia and breathy voice as complications of the injection yet, these complications were only encountered during the first week following injection and were entirely tolerated by the patients.

Botulinum toxin injection into the vocal folds in patients with bilateral abductor paralysis showed significant improvement of the respiratory symptoms both subjectively through the widening of the glottis gap, as noticed by video laryngoscopic examinations and objectively as demonstrated by the pulmonary function tests with minimal or no effect on voice or swallowing. Perhaps one limitation of this study is that, maybe we should have done a combined injection and denervation of both cricothyroid and thyroarytenoid muscles, both are tensors of the vocal fold, and their injection may lead to a more increase in the airway diameter thus a more pronounced improvement of the respiratory symptoms.

Further studies should be conducted to investigate the effectiveness of botulinum toxin injection on a larger sample of patients and to compare it with other methods of static enlargement of the airway.

\section{Conclusion:}

Chemical denervation of the thyroarytenoid muscle by botulinum toxin injection is a less invasive and a short-term treatment option available for improving airway obstruction in patients with BVFI with little or no effect on voice or swallowing.

\section{Declaration of interest:}

There are no interests to declare

\section{Financial support and sponsorship:}

No financial support was obtained from any source.

\section{Reference:}

1. Pinto JA, Godoy LB, Marquis VW, Sonego TB, Leal Cde F. Bilateral vocal fold immobility: diagnosis and treatment. Braz J Otorrhinolaryngol. 2011; 77(5):594-9.

2. Francis DO, Pearce EC, Ni S, Garrett CG, Penson DF. Epidemiology of vocal fold paralyses after total thyroidectomy for well-differentiated thyroid cancer in a Medicare population. Otolaryngol Head Neck Surg. 2014; 150: 548-57.

3. Goding G. Bilateral vocal fold immobility. In: Sulica L, Blitzer A, editors. Vocal Fold Paralysis. New York: Springer; 2006. P.237-48.

4. Lewis S, Woo P. Botulinum toxin in management of synkinesis in patients with unilateral and bilateral vocal fold paralysis. Laryngoscope. 2018; 128:447-50.

5. Blitzer A, Brin MF, Fahn S, Lange D, Lovelace RE. Botulinum toxin (BOTOX) for the treatment of "spastic dysphonia" as part of a trial of toxin injections for the treatment of other cranial dystonias. Laryngoscope. 1986; 96:1300-1.

6. Ekbom DC, Garrett CG, Yung KC, Johnson FL, Billante CR, Zealear DL, et al. Botulinum toxin injections for new onset bilateral vocal fold motion 
impairment in adults. Laryngoscope. 2010; 120:758-63.

7. Woo P, Mangaro M. Aberrant recurrent laryngeal nerve reinnervation as a cause of stridor and laryngospasm. Ann Otol Rhinol Laryngol. 2004; 113: 805-8.

8. Malki KH, Mesallam TA, Farahat M, Bukhari M, Murry T. Validation and cultural modification of Arabic voice handicap index. Eur Arch OtorhinoLaryngol. 2010; 267: 174351.

9. Kotby MN. The accent method of voice therapy. California: Singular ;1995. P. 3-21.

10.Miller MR, Hankinson J, Brusasco V, Burgos F, Casaburi R, Coates A, et al. Standardization of spirometry. Eur. Respir. J. 2005; 26, 319-38.

11.Zealear DL, Billante CR, Courey MS, Sant'Anna GD, Netterville JL. Electrically stimulated glottal opening combined with adductor muscle botox blockade restores both ventilation and voice in a patient with bilateral laryngeal paralysis. Ann Otol Rhinol Laryngol. 2002; 111:500-6.

12.Li Y, Pearce EC, Mainthia R, Athavale SM, Dang J, Ashmead DH, et al. Comparison of ventilation and voice outcomes between unilat?eral laryngeal pacing and unilateral cordotomy for the treatment of bilateral vocal fold paralysis. ORL J Otorhinolaryngol Relat Spec. 2013; 75:68-73.

13. Ongkasuwan J, Courey M. The role of botulinum toxin in the management of airway compromise due to bilateral vocal fold paralysis. Current opinion in otolaryngology \& head and neck surgery. 2011 Dec 1;19(6):444-8.

14.Daniel SJ, Cardona I. Cricothyroid onabotulinum toxin A injection to avert tracheostomy in bilateral vocal fold paralysis. JAMA OtolaryngologyHead \& Neck Surgery. 2014 Sep 1;140(9):867-9.

15.Andrade Filho PA, Rosen CA. Bilateral vocal fold paralysis: an unsual treatment with botulinum toxin. Journal of Voice. 2004 Jun 1;18(2):254-5. 